\title{
ESTIMATING THE LINK BETWEEN QUANTITATIVE EASING 3 AND BUSINESS INVESTMENT
}

\author{
Joseph Cheng and Julie Fitzpatrick
}

The BRC Academy Journal of Business 5, no. 1 (2015): 1-17.

http://dx.doi.org/10.15239/j.brcacadjb.2015.04.01.ja01

\section{WEB APPENDIX}

http://dx.doi.org/10.15239/j.brcacadjb.2015.04.01.wa01 


\section{Appendix 1}

Boskin (1978) estimated that consumption (C) declines by 1.06\% if interest rate (r) changes by $1 \%$ :

$$
\partial \operatorname{lnC} / \partial r=-1.06
$$

Recall that e in (14) represents the elasticity of demand for financial investment with respect to interest rate: $\mathrm{e}=\partial \mathrm{FT} / \partial \mathrm{r} \mathrm{r} / \mathrm{FT}$. Since all personal savings channel to financial investment, e is better known in economics literature as the elasticity of savings with respect to interest rate:

$$
\mathrm{e}=\partial \ln S / \partial \ln \mathrm{r}
$$

where

$$
\begin{gathered}
=\frac{\partial S / S}{\partial r / r}
\end{gathered}
$$

Since household savings (S) equal disposable income minus consumption, we can write

$$
\begin{gathered}
\partial S=-\partial C \\
=-\frac{\partial C / S}{\partial r / r} \\
=-\underline{\partial C} \underline{r} \\
\partial r S
\end{gathered}
$$

Dividing both the numerator and denominator by C:

$$
\begin{aligned}
& =-\underline{\partial \mathrm{C} / \mathrm{C}} \underline{\mathrm{r}} \underline{\mathrm{C}} \\
& \partial \mathrm{r} \quad \mathrm{S}
\end{aligned}
$$




$$
\begin{array}{cc}
=\frac{\partial \ln C}{r} & \underline{\mathrm{C}} \\
\partial \mathrm{r} & \mathrm{S}
\end{array}
$$

Thus,

$$
\mathrm{e}=-\partial \ln C / \partial r \quad \mathrm{r} C / \mathrm{S}
$$

For the 2013 data, if we use the average AAA and Treasury bond yields as a proxy of the interest rate (3.5\%) and we assume an average marginal tax rate of 30\%, then we find an after-tax yield of $2.43 \%$. Consumption and household savings in 2013 are \$11,537 Billion and \$618 Billion, respectively (Table 1). These values can be substituted into (19a) to estimate the value of e:

$$
\mathrm{e}=-(-1.06) \times .0243(11537 \mathrm{~B} / 618 \mathrm{~B})=0.48
$$


Table 1

\begin{tabular}{lrr} 
& Q3 2013 & Q3 2014 \\
\hline Households and institutions savings (\$ Billion) & 618.00 & 657.90 \\
Personal saving (\$ Billion) & 618.00 & 657.90 \\
Gross domestic investment (\$ Billion) & 3352.30 & 3481.70 \\
Personal Consumption Expenditures (PCEC) (\$ Billion) & 11537.70 & 11973.00 \\
New Security Issues, U.S. Corporations (\$ Billion) & 374.09 & 340.88 \\
New Issues, Treasury (\$ Billion) & 640.00 & 553.00 \\
Personal current taxes (\$ Billion) & 1657.80 & 1739.90 \\
Personal income (\$ Billion) & 14226.20 & 14801.20
\end{tabular}

Interest Rates (Average Yield)

$\begin{array}{lll}\text { Treasuries, July } & 2.44 & 2.40\end{array}$

$\begin{array}{lll}\text { Treasuries, August } & 2.42 & 2.40\end{array}$

$\begin{array}{lll}\text { Treasuries, September } & 2.43 & 2.40\end{array}$

$\begin{array}{lll}\text { Average Treasury (Q3) } & 2.43 & 2.40\end{array}$

$\begin{array}{lll}\text { Moody's Seasoned Corporate (AAA), July } & 4.34\end{array}$

$\begin{array}{lll}\text { Moody's Seasoned Corporate (AAA), August } & 4.54 & 4.08\end{array}$

$\begin{array}{lll}\text { Moody's Seasoned Corporate (AAA), September } & 4.64\end{array}$

$\begin{array}{lll}\text { Average Corp (AAA) (Q3) } & 4.51\end{array}$

$\begin{array}{lll}\text { Average of Treasury and Corporate (Q3) } & 3.47\end{array}$ 
e

E

Investment Realization Ratio
0.48

0.44

$-1.00 \quad-1.00$

0.87

0.90

Table 2

\begin{tabular}{lcr} 
E & Investment Realization Ratio & Estimated IB Increase for 2013 (\$ Billion) \\
\hline-0.10 & 0.40 & 410.91 \\
-0.20 & 0.57 & 585.82 \\
-0.30 & 0.67 & 682.69 \\
-0.40 & 0.73 & 744.22 \\
-0.50 & 0.77 & 786.76 \\
-0.60 & 0.80 & 817.93 \\
-0.70 & 0.83 & 841.75 \\
-0.80 & 0.84 & 860.55 \\
-0.90 & 0.86 & 875.76 \\
-1.00 & 0.87 & 888.33
\end{tabular}

